This is a postprint version of the following published document:

G. Prolongo, S., Cabanelas, J. C. \& Baselga, J. (2003). Reactive compatibilization of epoxy/ polyorganosiloxane blends. Macromolecular Symposia. Polymer Blends, 198 (1), pp. 283-293.

DOI: $10.1002 /$ masy.200350824

(C) Wiley, 2003 


\title{
Reactive Compatibilization of Epoxy/Polyorganosiloxane Blends
}

\author{
S. G. Prolongo, J. C. Cabanelas, J. Baselga* \\ Instituto Tecnológico de Química y Materiales Álvaro Alonso Barba. Universidad Carlos III de \\ Madrid. Avda. Universidad, 30, 28911 Leganés, Madrid, Spain \\ E-mail: jbaselga@ing.uc3m.es
}

\begin{abstract}
Summary:
A new thermoset material based on DGEBA with polyaminosiloxane curing agents is presented. The system shows reaction-induced compatibilization which prevents coalescence of polysiloxane and DGEBA rich domains, leading to gradient structured morphologies. The influence of curing temperature and/or chemical nature of the siloxane on the morphology and surface microhardness were examined. When siloxane is pre-reacted with epoxypropylphenylether (EPPE), a more homogeneous material is obtained. Microhardness profiles on the material are strongly influenced by the extension of the compositional gradients.
\end{abstract}

Keywords:

blends, epoxy resin, poly(3-aminopropylmethylsiloxane), reactive compatibilization, thermosets

\section{Introduction}

Fracture toughness and impact resistance of highly crosslinked systems, as epoxy resins, are usually low limiting the use of these materials in applications requiring high fracture strength. The problem is usually solved introducing elastomeric (ATBN, CTBN) and/or thermoplastic (PSF, PEI, PPO, PMMA) materials, which form a segregated phase in the cured state, improving the mechanical properties of the blend. ${ }^{[1]}$

Polysiloxanes have very low glass transition temperature and good thermal stability, and may be used as epoxy toughening agents, especially for high temperature applications. Since they are immiscible with epoxy resins, different compatibilization approaches, including copolymer formation and reactive end groups insertion, have been proposed in literature. ${ }^{[2-5]}$

In this work, we follow a new and different approach. We have used a highly functionalised organosiloxane polymer, poly(3-aminopropylmethyl-siloxane) (PAMS), as a hardener for a 
DGEBA base resin. The blend is completely immiscible at room temperature, but from our early studies, ${ }^{[6,7]}$ it becomes partially miscible as polymerisation proceeds, due to the high functionality of the PAMS. A careful selection of the curing temperature allows tuning different final morphologies ranging from macroscopically homogeneous transparent samples to partially phase segregated opaque samples. This new system has some good properties like low water absorption, good adhesion and transparency but it is relatively brittle. In addition, curing reaction rate is fast and similar to that of DGEBA with other aliphatic amines and does not seem to be influenced by the low conversion gelation process.

The definition of homogeneity in thermosets is not completely closed. It is well known that it is impossible to reach complete conversion; if heterogeneity is defined in a low enough scale, cured resins would present intrinsic heterogeneities. If the monomers are immiscible, as in the present case, the increase of viscosity and crosslinking degree during cure should limit the diffusion controlled processes and severe concentration gradients should be observed. The objective of this work consists of the characterization of the microscopic homogeneity and to study the influence of the polysiloxane chemical composition on it. The polysiloxane functionality and the lateral chain composition is modified pre-reacting the amine groups with epoxypropylphenylether. Semi-quantitative SEM X-ray analysis will be used to analyse the presence of concentration gradients. The observed microstructures will be correlated with the microhardness of the different cured products.

\section{Experimental}

Materials. Diglycidyl ether of bisphenol-A based resin (DGEBA) was supplied by Gairesa (Spain) with an epoxy equivalent of $188 \mathrm{~g}$ (equiv. epoxy) ${ }^{-1}$, as determined by acid titration. The ratio of secondary hydroxyl groups to epoxy group was 0.069 as determined by size exclusion chromatography (SEC) (Shimadzu LC9A). Poly(3-aminopropylmethylsiloxane) (PAMS) was synthesised from 3-aminopropylmethyldiethoxysilane monomer (from ABCR) by hydrolysis and condensation reaction. ${ }^{[6]}$ The oligomer was characterized by SEC, vapour pressure osmometry (VPO, Knauer) and ${ }^{29} \mathrm{Si}$ NMR spectroscopy (Gemini). Number-average molecular weight measured by VPO was $1620 \pm 90 \mathrm{~g} \mathrm{~mol}^{-1}$ and $1570 \mathrm{~g} \mathrm{~mol}^{-1}$ by ${ }^{29} \mathrm{Si} \mathrm{NMR}$, with an amine equivalent of $58.5 \mathrm{~g}$ (equiv. active hydrogen) $)^{-1}$. Tg of PAMS was $-65 \pm 2^{\circ} \mathrm{C}$ (DSC, Perkin Elmer, $10 \mathrm{~K} \mathrm{~min}^{-1}$, midpoint). 
Lateral chain modification of PAMS was accomplished by reaction of its amine groups with 2,3epoxypropylphenylether (EPPE) at different molar ratios. Reactions were carried out in a flask with continuous stirring at $333 \mathrm{~K}$ for 3 hours under $\mathrm{N}_{2}$ atmosphere. Table 1 shows the characteristics of samples prepared with different PAMS/EPPE ratios. The progress of the lateral reaction was checked following the extinction of the primary amine band $\left(4936 \mathrm{~cm}^{-1}\right)$ in the near IR (Perkin-Elmer GX2000, $4 \mathrm{~cm}^{-1}$ resolution). Tg of the samples was also analysed by DSC.

Table 1. Characteristics of the polysiloxane hardeners.

\begin{tabular}{cccc} 
Sample & $\mathrm{Mn}$ & $\mathrm{r}_{\mathrm{EPPE}}{ }^{\mathrm{a})}$ & $\mathrm{T}_{\mathrm{g}}$ \\
\cline { 2 - 4 } & $\mathrm{G} / \mathrm{mol}$ & & ${ }^{\circ} \mathrm{C}$ \\
\hline PAMS & 1600 & 0 & -67 \\
PAMS-EP10 & 1805 & 10.2 & -54.6 \\
PAMS-EP20 & 2010 & 20.0 & -39.8 \\
PAMS-EP40 & 2420 & 40.4 & -18.8 \\
\hline
\end{tabular}

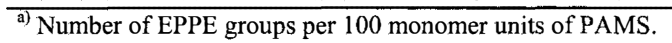

Sample preparation and curing. Stoichiometric DGEBA and PAMS / PAMS-EPPE mixtures were prepared at room temperature and placed in a mould between two glass slides separated by a Teflon sheet, which were pre-treated with a demoulding agent. Curing was performed in an oven for 2 hours at different temperatures, as it is shown in Table 2. Tg of cured samples were obtained from DMTA measurements (Rheometric Scientific MkIII), using bars of $10 \times 5 \times 1 \mathrm{~mm}$ that were tested in bending mode at $1 \mathrm{~Hz}$ and $2 \mathrm{Kmin}^{-1}$ heating rate. Tg was determined as the temperature at the maximum in a $\tan \delta$ scan. Curing was checked by FT-NIR (Perkin-Elmer GX2000) following the area of the epoxy band centered at $4530 \mathrm{~cm}^{-1}$.

Determination of morphology. A Labophot microscope (Nikon) provided with photographic camera was used for transmission optical microscopy (TOM) and epifluorescence microscopy (EFM) imaging observations of the initial mixtures. Objectives of $\times 10$ and $\times 40$ with numeric apertures of 0.25 and 0.65 , respectively, were used. For fluorescent labelling, 1,5dimethylaminonaphtalen-5-sulphonylchloride (dansyl chloride, DNS; Molecular Probes) was added to PAMS (1:5000 molar ratio) and heated at $60{ }^{\circ} \mathrm{C}$ for $16 \mathrm{~h}$. Reaction between DNS and PAMS was complete as checked by thin layer chromatography (TLC). Chromophore concentration was $10^{-4} \mathrm{M}$, as determined by UV/VIS spectrophotometry. DNS labelled PAMS 
was stored in a desiccator preserved from the light. EFM observations were taken with a barrier filter $\left(\lambda_{\max }<420 \mathrm{~nm}\right)$.

Morphology of cured material was analysed measuring the X-ray signal of silicon atoms on $\mathrm{Au} / \mathrm{Pd}$-coated samples in an XL-30 SEM microscope (Phillips). X-ray semi-quantitative microanalysis was performed with an EDAX DX4i detector. Surface was analysed by mapping a $15 \times 15$ point matrix over a surface of $2 \mathrm{~mm}^{2}$.

Microhardness. Vickers microhardness (square-based diamond pyramid) measurements were performed using a Micro Hardness tester DMT HVS-1000 (Time Technology), which allows displacement of the indenter along the x-length. Two different loads were employed: $2 \mathrm{~N}$ for indentations of about $0.2 \mathrm{~mm}^{2}$ area, and $0.25 \mathrm{~N}$ for indentations of about $4.510^{-4} \mathrm{~mm}^{2}$. Measurements with the low load were carried out every $40 \mu \mathrm{m}$, over an axis-length of $1 \mathrm{~mm}$, totalling 25 consecutive measurements.

Table 2. Epoxy conversion and glass transition of cured samples.

\begin{tabular}{ccccc} 
Code & Hardener & $\begin{array}{c}\text { Curing temp } \\
\left({ }^{\circ} \mathrm{C}\right)\end{array}$ & $\begin{array}{c}\text { Epoxy } \\
\text { conversion }\end{array}$ & $\begin{array}{c}\mathrm{Tg}^{\mathrm{a}} \\
\left({ }^{\circ} \mathrm{C}\right)\end{array}$ \\
\hline C60 & PAMS & 60 & 0.86 & 98 \\
C80 & PAMS & 80 & 0.88 & - \\
C100 & PAMS & 100 & 0.93 & 155 \\
C120 & PAMS & 120 & 0.96 & - \\
C150 & PAMS & 150 & 0.97 & 163 \\
\hline C60P20 & PAMS-EP20 & 60 & 0.81 & 90 \\
C120P20 & PAMS-EP20 & 120 & 0.86 & 109 \\
C150P20 & PAMS-EP20 & 150 & 0.92 & 118 \\
\hline C120P10 & PAMS-EP10 & 120 & 0.96 & 125 \\
C120P40 & PAMS-EP40 & 120 & 0.74 & 73 \\
\hline a) & & & &
\end{tabular}

a) DMTA

b) This sample presents a shoulder at $110^{\circ} \mathrm{C}$

\section{Results and Discussion}

Curing. From an inspection of data presented on Table 2, epoxy conversion increases with curing temperature up to $150^{\circ} \mathrm{C}$, at which the system reaches the maximum conversion. The high value of the Tg for DGEBA/PAMS samples reflects the high crosslinking density due to the high PAMS functionality; this point is especially notable if take into account the very flexible chain hardener used. When PAMS is pre-reacted with EPPE the initial mixtures with DGEBA remain also immiscible. The measured $\mathrm{Tg}$ of the pre-reacted systems is lower than the Tg of DGEBA/PAMS 
systems cured at the same conditions, as a consequence of the decrease in functionality of the modified PAMS. Samples with the same epoxy conversion show also lower Tg if they are prereacted with EPPE and the resulting decrease in crosslinking degree.. It is noticeable that the sample with the highest EPPE content, PAMS-EP40, showed a high temperature shoulder (at around $110^{\circ} \mathrm{C}$ ) in the $\tan \delta \mathrm{scan}$; it should be expected a highly heterogeneous microstructure for this sample.

Morphology development during curing. Room temperature mixtures have cloudy appearance in all the studied systems showing that the components are not miscible. A brief analysis of the initial morphology was done on DGEBA/PAMS mixtures immediately after mixing. TOM image just after mixing is presented in Figure 1a. Two phases are clearly observed. The dispersed phase forms quasi-spherical domains of about $2-5 \mu \mathrm{m}$ surrounded by the continuous phase. Figure $1 \mathrm{~b}$ shows the same region by epifluorescence microscopy (EFM). Bright areas correspond to the blue emission of DNS $\left(\lambda_{\max } \approx 460 \mathrm{~nm}\right.$ ). Since DNS was only attached to PAMS, these areas belong to PAMS-rich domains. PAMS constitutes therefore the continuous phase.

a)

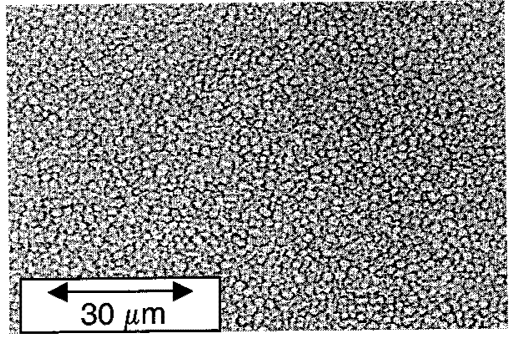

b)

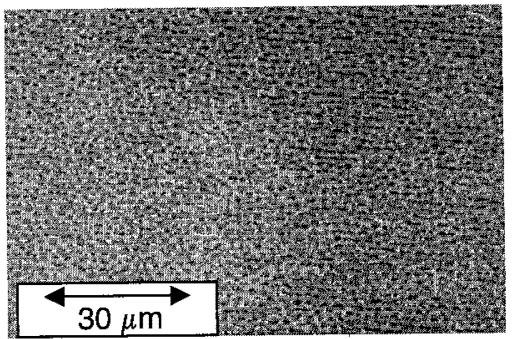

Fig. 1. a) TOM image of the DGEBA/PAMS mixture at room temperature. b) The same area observed by epifluorescence microscopy (EFM).

Sample is then heated for 2 minutes at $60^{\circ} \mathrm{C}$ and then cooled down again to room temperature. The resulting morphology is presented in Figure 2a. At this time, conversion is very low $(\alpha<0.05)$ and main species are partially reacted PAMS with DGEBA and pure DGEBA; tertiary amine concentration is negligible at this conversion and so the crosslinking degree. A coalescence phenomena and phase inversion are observed. The dispersed phase size now is high but, as deduced from the EFM image (Figure 2b), it is composed mainly by PAMS. The polysiloxane is now the dispersed phase in an epoxy-rich (low fluorescence) continuous phase. A clear 
explanation for this behaviour is not available at the moment but it seems that the driving force may rely on the high functionality of PAMS. According to condensation reaction statistics ${ }^{[8,9]}$, despite the low conversion reached, the number of non-reacted "free" PAMS molecules is now very low, becoming the minority component of the mixture.

a)
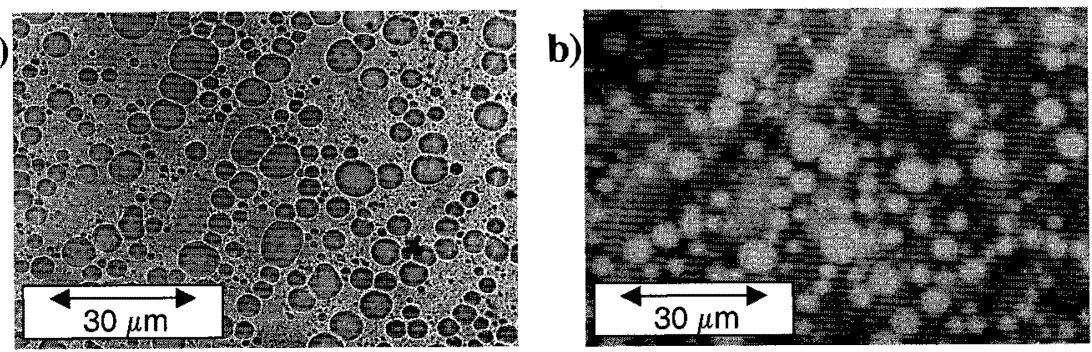

Fig. 2. a) TOM image of the DGEBA/PAMS mixture quenched at room temperature after $2 \mathrm{~min}$. at $60^{\circ} \mathrm{C}$. b) The same area observed by epifluorescence microscopy (EFM).

Observation of the morphology of cured samples by TOM or EFM is not easy because the refractive index of the components in the cured system are very similar to resolve the images by TOM, and because there are no clear phase boundaries to obtain good contrast by EFM. Since silicon atoms are only present in the hardener component, PAMS, we have performed a semiquantitative analysis of the silicon surface distribution to study PAMS distribution in the cured specimens. Due to the uncertainty in the penetration depth of the electron beam and the lack of a Si standard in a similar matrix, it is not possible to give absolute values of $\mathrm{Si}$ concentration. For quantification purposes, the signal obtained in each of the 225 matrix points was normalized with the average Si signal measured in the total scanned area.

Figure 3 shows the concentration contour graphs for silicon distribution in the DGEBA/PAMS blend for three curing temperatures. A gradient structure is observed, especially for low conversion degrees, in which Si-rich domains of about 100-150 $\mu \mathrm{m}$ in size can be observed. With respect to the initial room temperature morphology, it can be observed that PAMS severely coalesces. Since the scanned area is large enough, it can be assumed that the surface average $\mathrm{Si}$ signal is proportional to the bulk $\mathrm{Si}$ concentration. Therefore, for sample cured at $80^{\circ} \mathrm{C}$ (sample with highest compositional heterogeneity) the PAMS:DGEBA equivalent ratio in the domains of highest PAMS concentration is about $4: 1$, and about $1: 2$ for the regions were PAMS 
concentration is minimal. For samples cured at higher temperatures, compositional heterogeneity is lower but the same morphology remains (figure 3b). Modification of the lateral composition of PAMS with EPPE should have the benefit of increasing initial miscibility with DGEBA.
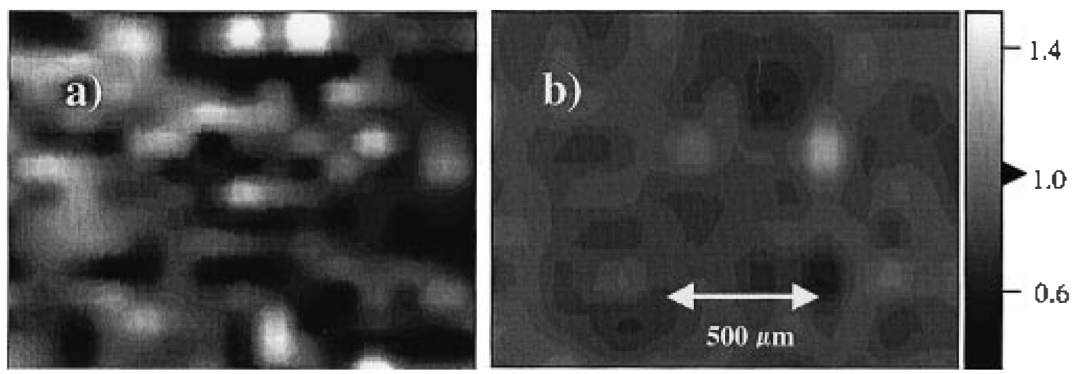

Fig 3. Silicon concentration mapping of the surface of stoichiometric DGEBA/PAMS samples and cured at a) $80^{\circ} \mathrm{C}$, b) $150^{\circ} \mathrm{C}(2 \mathrm{~h})$. Grey scale corresponds to normalized Si signal.

In Figure 4 the same concentration map is presented for the DGEBA/PAMS-EP10 blend. Using PAMS pre-reacted with EPPE as hardener, concentration gradients seem to be smaller, even for the lower conversions, but surface morphology does not change appreciably. Coalescence of PAMS was not avoided
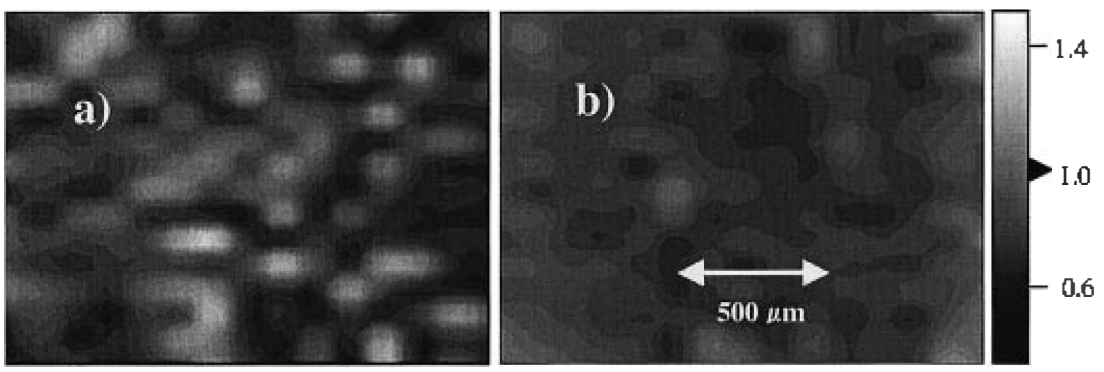

Fig. 4. Silicon concentration mapping of the surface for stoichiometric DGEBA/PAMS-EP10 samples and cured at a) $60^{\circ} \mathrm{C}$, b) $150^{\circ} \mathrm{C}(2 \mathrm{~h})$. Grey scale corresponds to normalized Si signal.

Data presented in Figures 3 and 4 needs to be quantified. We have divided the silicon concentration values into classes. The probability to find a region with the silicon concentration of 
the $n$ class is defined by $p(i)=A(i) / \sum_{j=1}^{n} A(j)$ where $A(i)$ is the area occupied by the range of silicon concentration from the $\mathrm{n}^{\text {th }}$ class and the sum corresponds with the total area analyzed. In Figure 5 we present the probability function for $\mathrm{Si}$ distribution for the cured samples and in Table 3, the distribution function parameters are presented. It can be observed a Gaussian like distribution curve around the mean silicon concentration. The distribution sharpens as curing degree increases.

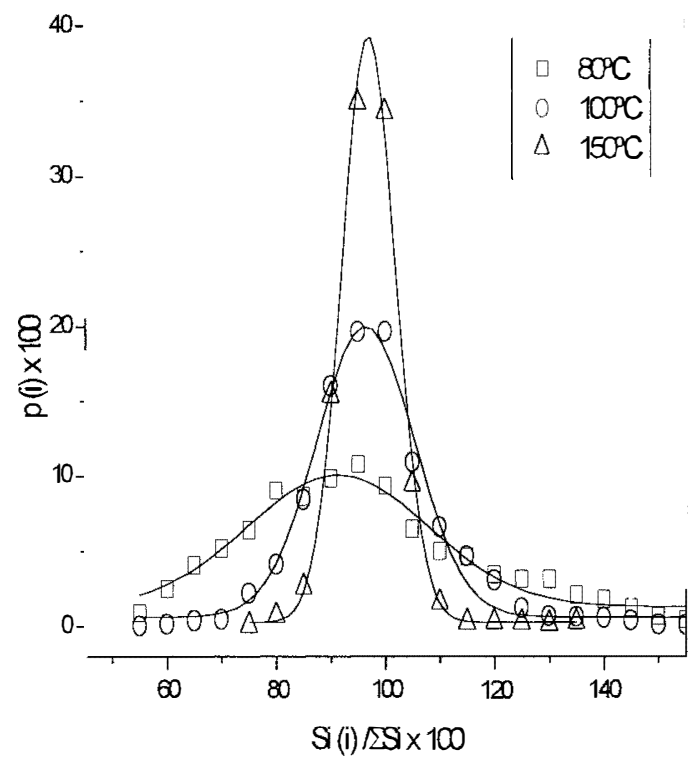

Fig. 5. Si distribution function for the DGEBA/PAMS system at different curing temperatures.

Several important conclusions can be obtained from Table 3 and Figure 5 It becomes evident that as curing degree increases samples become more homogeneous, but the analysis of the standard deviation reflects an interesting feature. Samples cured with PAMS containing 20\% of EPPE pending units are more homogeneous, with sharp and symmetric distribution functions despite the lower conversions achieved. For the sample with 40\% EPPE content. which presented a high temperature shoulder in the $\tan \delta$ scan, a very wide standard dev ration is observed suggesting a 
bimodal $\mathrm{Si}$ distribution. These results allow speculating about the morphology evolution. Coalescence and curing seem to be two simultaneous competing processes for fixing microstructure. At low temperatures, curing rate is low enough and coalescence of PAMS creates big domains. At higher temperatures, crosslinking rate limits the coalescing process resulting in a more homogenous microstructure.

Table 3. Statistical analysis of the cured samples.

\begin{tabular}{cccc}
\hline Sample & Conversion & Standard Deviation $^{\text {a) }}$ & Asymmetry \\
\cline { 3 - 4 } C80 & 0.88 & 22.1 & 0.62 \\
$\mathrm{C} 100$ & 0.93 & 12.7 & 0.93 \\
$\mathrm{C} 150$ & 0.97 & 6.0 & 1.08 \\
\hline $\mathrm{C} 60 \mathrm{P} 20$ & 0.81 & 13.8 & 0.90 \\
$\mathrm{C} 120 \mathrm{P} 20$ & 0.86 & 11.4 & 0.98 \\
$\mathrm{C} 150 \mathrm{P} 20$ & 0.92 & 7.4 & 1.04 \\
\hline $\mathrm{C} 120 \mathrm{P} 10$ & 0.96 & 9.9 & 1.02 \\
$\mathrm{C} 120 \mathrm{P} 40$ & 0.74 & 43.9 & 0.22 \\
\hline a) Over a mean Si concentration normalized to 100. &
\end{tabular}

a) Over a mean $\mathrm{Si}$ concentration normalized to 100 .

Table 4. Vickers Hardness (MPa) of DGEBA/PAMS cured resins.

\begin{tabular}{lcccc}
\hline \multirow{2}{*}{ Sample } & \multicolumn{4}{c}{ Curing T $\left({ }^{\circ} \mathrm{C}\right)$} \\
\cline { 2 - 5 } & 60 & 80 & 100 & 120 \\
DGEBA/PAMS & $176 \pm 2$ & $164 \pm 1$ & $155 \pm 5$ & $152 \pm 3$ \\
DGEBA/PAMS-EP10 & $194 \pm 4$ & $185 \pm 3$ & $173 \pm 2$ & $161 \pm 3$ \\
DGEBA/PAMS-EP20 & $196 \pm 4$ & $191 \pm 3$ & $173 \pm 3$ & $161 \pm 2$ \\
DGEBA/PAMS-EP40 & $124 \pm 5$ & $144 \pm 4$ & $146 \pm 3$ & $149 \pm 3$ \\
\hline
\end{tabular}

Hardness measurements. In Table 4 the overall Vickers hardness is presented. The values presented are similar to literature data for epoxy novolacs and other brittle resins. ${ }^{[10,11]}$ Hardness data for DGEBA/PAMS blends show a clear correlation with curing temperature being lower as curing (conversion) increases probably due to an increase in brittleness. It is interesting to note the behaviour for EPPE pre-reacted systems. For these systems, hardness is systematically higher than for pure PAMS systems but its variation with curing temperature shows the same trend.

Since hardness is a surface property hardness data may reveal the presence of depth heterogeneity which accumulates to the two-dimensional surface heterogeneity Micro-hardness measurements were made along and axis and results are displayed in Figure 6. The values observed fluctuate around the mean hardness and the only explanation is the heterogeneous distribution of epoxy and 
hardener over the surface Peak to peak distances range from 50 to $100 . \mu \mathrm{m}$, comparable to the PAMS-rich domain size observed by SEM X-ray analysis The fluctuations are in the order of $\pm 20 \%$ for the lowest cured sample, and become small as curng and homogeneity increases. confirming the importance of these heterogeneities on the mechanical behaviour of the material. $S_{1}$ content was analysed in each of the impressions and no clear correlation was found between $\mathrm{S}_{1}$ content and micro-hardness

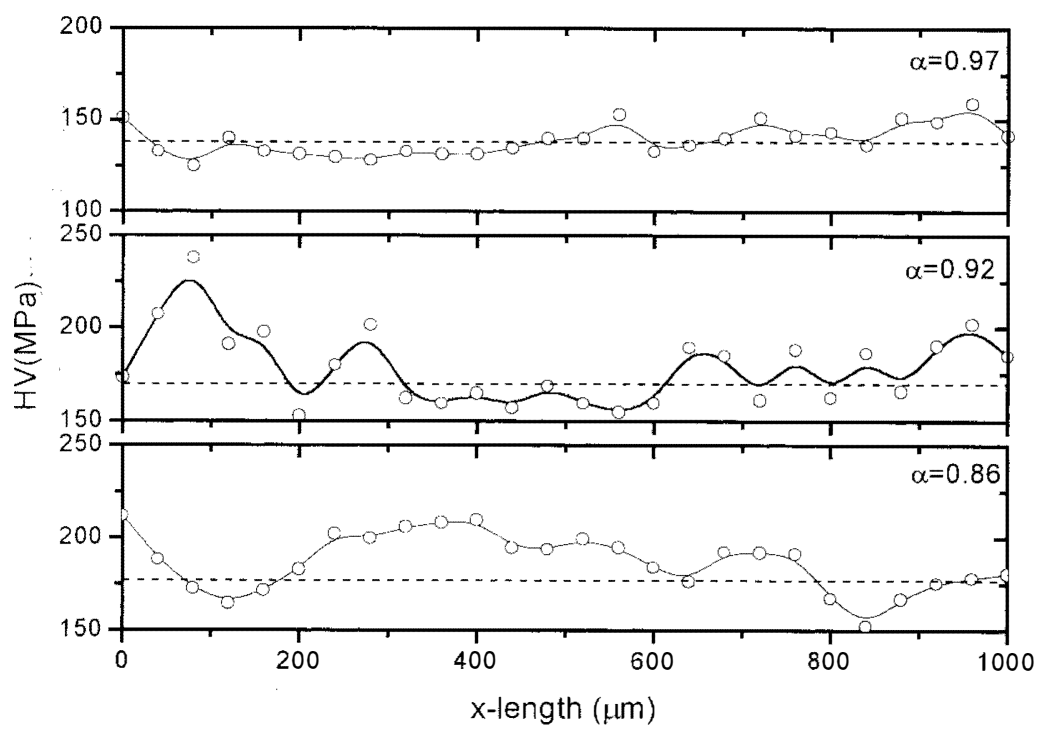

Fig. 6. Micro-hardness profiles in function of curing degree for DGEBA/PAMS samples. Mean hardness is also presented by an horizontal dot line.

\section{Conclusions}

The morphologv and surface properties of a new epoxy, silicone system based in a polyfunctional aminosiloxane (PAMS) hardener have been studied. These components are immıscible at room temperature and thev are initially phase separated. At curıng temperature, PAMS coalesce forming large domains and as reaction proceeds a gradient structure is formed with PAMS and DGEBA rich "domans", but no phase boundaries are present Quantification of these domains in cured resin was possible by using SEM X-rav microanalysis Because of the competition between two 
simultaneous phenomena, curing and coalescence, cured resins show different heterogeneity as function of curing degrees, being more homogeneous at the highest curing temperatures employed. Homogeneity can be improved if lateral chemical composition of PAMS is varied prereacting with a monoepoxide monomer. PAMS becomes more compatible with DGEBA, but final $\mathrm{Tg}$ falls off because of the drop in crosslinking density Microhardness measurements revealed the presence of a gradient structure and the length of the domain sizes coincides with SEM observations.

[1] D.R. Paul, C.B. Bucknall, "Polymer Blends", Wiley Interscience,New York 2000, chap. 13, 22 and 26.

[2] T. Kasemura, S. Takahashi, K. Nishihara, C. Komatu, Polymer 1993, 34, 3416.

[3] S. Hou, Y. Chung, C. Chan, P. Kuo, Polymer 2000, 41, 3263.

[4] J. L. Hedrick, T. P. Russell, B. Haidar, A.C.M. Yang, Macromolecules 1989, 22, 4470.

[5] M. Ochi, Polymer 1999, 40, 1305.

[6] J.C. Cabanelas, B. Serrano, J. Gonzalez-Benito, J. Bravo, I. Baselga, Macromol. Rapid. Comun 2001, 22694

[7] J.C. Cabanelas, S.G. Prolongo, B. Serrano, J. Bravo, J. Baselga, J. Mat. Process. Tech. 2002, to be published.

[8] W.H. Stockmayer, J. Polym. Sci 1952, 9, 69

[9] W.H. Stockmayer, J. Polym. Sci 1953, 11, 424.

[10] M. Krumova, C. Klingshirn, F. Haupert, K. Friedrich, Compos. Sci. Techol. 2001, 61, 557

[11] D.A. Tilbrook, R.L. Clarke, N.E. Howle, M. Braden, Biomaterials 2001, 21, 1743. 